\title{
Interactive comment on "A semi-continuous study on the toxicity of atmospheric particles using a versatile aerosol concentration enrichment system (VACES): development and field characterization" by Xiaona Shang et al.
}

\section{Anonymous Referee \#2}

Received and published: 4 December 2020

The manuscript represents the results obtained by using a versatile aerosol concentration enrichment system (VACES), which was extended in order to estimate the toxicity of ambient air. The differences between concentrated and non-concentrated particulate matter were manifested in the measured aerosol properties; while offline and online sampling equipment has been applied to investigate in detail the acquired toxicity data. The observed results were further enhanced by studying the correlations between the measured toxicity and all obtained physical/chemical aerosol parameters, in order to highlight the role of aerosol enrichment through the VACES technique. Overall, 
the results are interesting and well investigated but the manuscript is difficult to follow. An important vantage of this study is that it includes a variety of techniques, providing several aerosol properties that may capture the effect of VACES on sampled particulate matter. Finally, it is essential that this study attempts to highlight the role of aerosol chemical components on the measured toxicity levels. Nevertheless, the manuscript needs some major revision regarding the selected toxicity assay, the fact that the utilized techniques are not clearly described and, in several places the justifications or discussions should be presented in a more comprehensive way.

Some indicative major issues to be addressed are the following: Section 1: Please justify the application of photobacteria in atmospheric particle toxicity and its association with health effects. Section 2.2: It is difficult to follow the description and the setup of the instrumentation. Please rephrase to clarify the definition of the optimal technical parameters, the calculation of measured theoretical concentrations, the utility of ambient aerosol removal through filter, the description of performance testing, the essential technical difference between offline and online collection, the justification of selected sampling parameters (both offline and online). Please rephrase to describe the obtained samples since there are collections mentioned as continuous, based on $1 \mathrm{~min}$, on $8 \mathrm{hrs}$, on $1 \mathrm{hr}$ and on 30min. Section 2.3: As already mentioned in Section 1 , the selected toxicity assay is mentioned in Jang et al., (2019) as a biotoxic assay based on a luminescent bacterium. Please justify the selection of this particular assay and clarify how this could be linked to health effects. Please mention whether this assay has been part of epidemiological studies or has been compared / combined with any cellular or acellular aerosol toxicity assay linked to health effects. Section 3.3: The results should be clearly described and justified in a comprehensive way. For example, please rephrase to clarify clean and polluted days, meaning of toxicity of non-ambient samples, normal fluctuation range of the luminescence bacterium affecting the sample uncertainty, effect of low toxicity of ambient samples on concentration, differences in variability between EFs and ambient and VACES samples, effect of enrichment in chemical composition of samples. Please note that it would be helpful to add a more

Interactive comment
Printer-friendly version

Discussion paper 
detailed description in the legends of the figures, regarding the properties of the presented samples.

AMTD

Interactive comment on Atmos. Meas. Tech. Discuss., doi:10.5194/amt-2020-10, 2020.

Interactive comment 